\title{
Myelinated nerve fiber layer associated with other ocular pathology in a 20-year-old myopic man
}

\author{
Jovanovic Dragan*, Ruszkowski Alissa \\ Trinity School of Medicine, Alphareta, GA, USA, Saint Vincent and the Grenadines Campus
}

${ }^{\star}$ Corresponding author: Jovanovic Dragan, Trinity School of Medicine, Alphareta, GA, USA Saint Vincent and The Grenadines Campus; E-mail: djovanovic@ trinityschoolofmedicine.org

Received: June 25, 2019; Accepted: July 04, 2019; Published: July 09, 2019;

\begin{abstract}
Background: Myelinated retinal nerve fibers (MRNFs) are usually unilateral and asymptomatic benign lesions associated with mild hypermetropia, emmetropia, or severe myopia. We describe herein an myopic patient with a syndrome of ipsilateral myelinated retinal nerve fibers associated with other pathological changes: epiretinal membrane with pseudohole, vitreomacular traction syndrome and bilateral lattice degeneration.

Methods: Extended ophthalmoscopy was used to evaluate retinal posterior segment. Infrared reflectance imaging helped in visualizing sub-retinal pathology. Detailed images from within the retina were obtained by using optical coherence tomography.

Results: Ophthalmologic examination revealed ipsilateral small temporal myopic conus with lattice degeneration temporally and inferotemporally. The area of myelinated nerve fiber extended into the pappilomacular bundle, but did not reach the fovea. At the fovea, an epiretinal membrane with clinically visible vitreomacular traction and some retinal elevation was found. In the peripheral fundus areas of lattice degeneration temporally and inferiorly was also noted. The OCT scans of the affected eye showed an incomplete posterior vitreous detachment with traction of a thickened posterior hyaloid base to the fovea. There was distortion of the foveal anatomy with a small amount of subfoveal fluid but a full-thickness macular hole could not be detected. Macular traction detachment of retina and epiretinal membrane with macular hole were treated surgically (S/P Repair Complex RD (25g, 20\% SF6, membrane peel with gas endotamponade) and vitreomacular traction was released. Lattice degeneration was treated by laser (S/P Laser).
\end{abstract}

Conclusions: MRNF lesions were stable and although associated with other ocular pathology progression was not observed during follow-up.

Keywords: Myelinated - Myopia - Lattice degeneration - Vitreomacular traction - Coherence tomography

\section{Introduction}

Myelinated retinal nerve fibers (MRNFs) are usually unilateral and asymptomatic benign lesions of the retina around the optic disk [1-2]. but it could also be found in other parts of the retina and in fovea [3]. During embryonic development the retinal nerve fibers may retain the myelin coat resulting in abnormal intraocular myelination of the peripheral nerve anterior to the lamina cribrosa [4]. The MRNF may be inherited but early-age trauma to the eye damaging lamina cribrosa may let oligodendrocytes to pass to the retina causing myelination [5]. MRNF has been associated with mild hypermetropia, emmetropia, or severe myopia. The size and the location of opaque nerve fiber patch determine visual field defects in eyes with MRNF [2], [6]. Studying the correlation between the extend of myelinated nerve fibers and refraction anomalies Schmidt D. at al [7]. concluded that myopia only occurred in eyes with wide-spread myelinated nerve fibers but not in eyes with circumscribed myelinated nerve fibers.

\section{Case report}

A 20-year-old man presented with severe left eye (OS) central blurred vision worsening during a period of one year. Reading and watching TV were affected activity. On the right eye (OR) he had gradual onset of blurred vision with mildly affected reading. The patient was noted to have an area of myelinated nerve fiber layer in his left eye a number of years ago, which remained stable over several years of follow-up. More recently change in visual acuity of the left eye was noted. The patient's past ocular history was also notable for anisometropia, but he did not report a history of amblyopia. On examination visual acuity with correction at distance measured 20/25 OD and 20/80 OS. RO examination also revealed a small temporal myopic conus and lattice degeneration temporally - inferotemporally and a few additional areas inferiorly (Fig.1A). Optical Coherence Tomography (OCT) showed normal thickened retinal nerve fibers with an attached hyaloid (Fig.1B). Left eye fundus examination showed abnormal layer of blood vessels and circumferential areas of lattice degeneration temporally and inferotemporally (Fig.1C). The area of myelinated nerve fiber extended into the papillomacular bundle, but did not reach the fovea. At the fovea, an epiretinal membrane with clinically visible vitreomacular traction and some retinal elevation was noted. The OCT scans of the left eye showed an incomplete posterior vitreous detachment with traction of a thickened posterior hyaloid base to the fovea distorting foveal anatomy with a small amount of subfoveal fluid but a full-thickness macular hole could not be detected (Fig. 1D). Myelinated nerve fiber lesions showed hyperreflectibility 
(Fig. 2). Autofluorescence imaging revealed a dark area in the region of myelinated RNFL (Fig. 3). Macular traction detachment of retina and epiretinal membrane with macular hole were treated surgically (S/P Repair Complex RD (25g, 20\% SF6, membrane peel with gas endotamponade) and vitreomacular traction was released. Lattice degeneration was treated by laser (S/P Laser). Initial ocular post-op medication was: Cyclogyl 2\%, Gentacidin 0.3\%, and Prednisolone Acetate $1 \%$. Three days after surgery Timol (Maleate) $0.25 \%$ was added. Retinal examination found periphery laser scarring and lattice degeneration with atrophic hole with laser (inferotemporal). The patient was regularly followed up in order to monitor progression and one year after surgery no disk edema and pallor, no new holes or tears were found and retinal vessels had normal caliber.
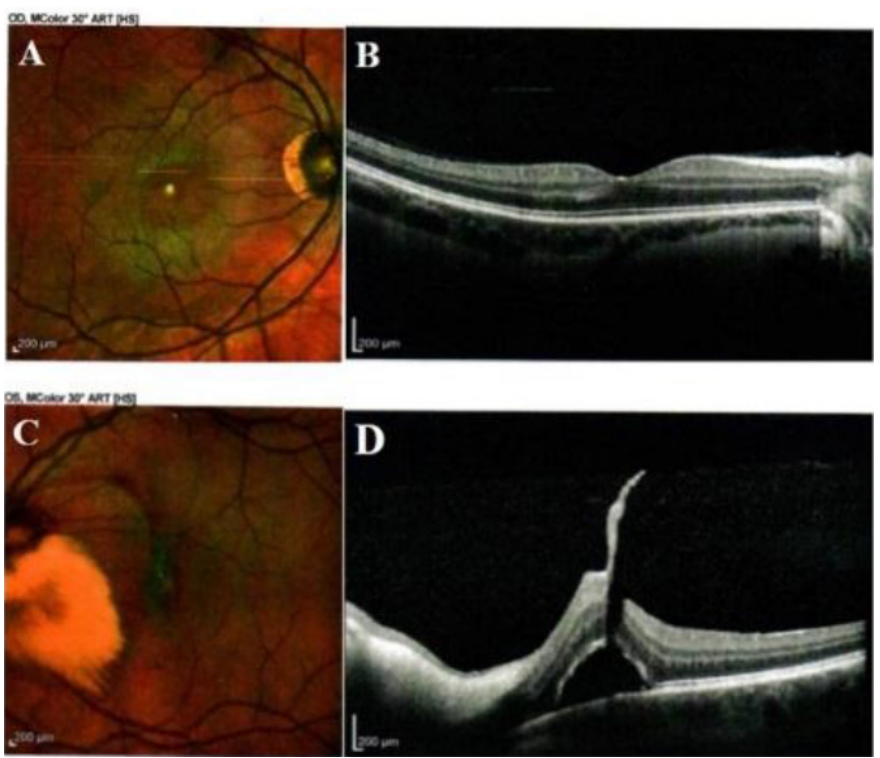

Figure 1. Optic disc imaging findings: A, OD color fundus photograph showing circumferential areas of lattice degeneration emporally - inferotemporally. B, OD OCT showing normal thickened retinal nerve fibers with an attached hyaloid . C, OS color fundus photograph showing a large area of MNFL extended into the papillomacular bundle without reaching the fovea. $\mathbf{D}$, OS OCT showing an incomplete posterior vitreous detachment with traction of a thickened posterior hyaloid base to the fovea. Distorted foveal anatomy with a small amount of subvoveal subretinal fluid.

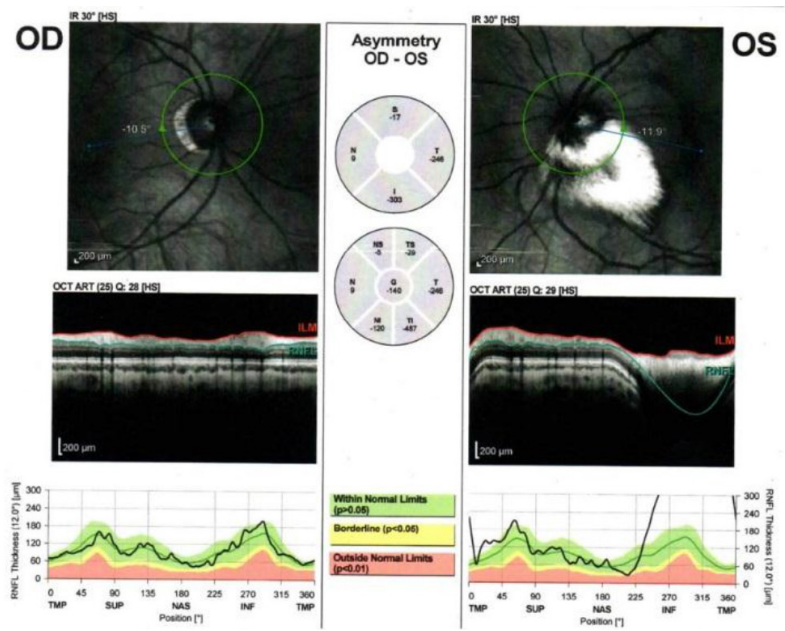

Figure 2. OCT of the right and of the left eye showed hyperreflectivity of myelinated nerve fiber lesions in the left eye.
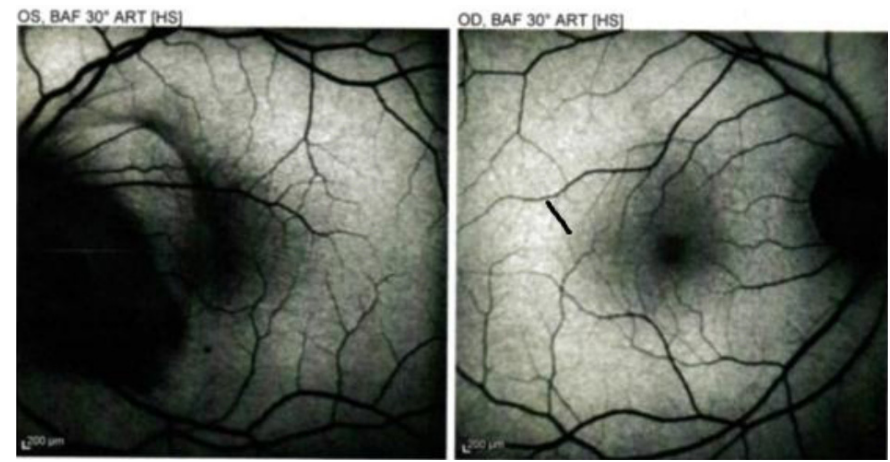

Figure 3. Autofluorescence imaging reveals a dark area in the region of the myelinated RNFL in OS.

\section{Discussion}

MNFL represent an asymptomatic developmental anomaly in which myelin sheaths extend to retinal nerve fibers along their intraocular portion causing displacement of the axons toward the vitreous body causing decreased vessel density in MRNF areas [8]. In our patient MRNF lesions were stable and although associated with other ocular pathology progression was not observed during followup. Cases with regression of MRNF associated with inflammatory diseases and glaucoma were also reported in the literature [9], [10]. Our patients was myopic (OD - 10.25 and OS - 5.50) and ophthalmologic examination revealed a small temporal myopic conus in right eye. High myopia is one of the leading causes of low vision in the world. [11] Genetic and environmental factors play role in its development [12]. Physiological myopia is a common optical aberration [13], but in pathological shortness with irreversible conditions such as retinal detachment, and macular atrophy can lead to blindness. In the myopic eye excessive axial elongation can lead to mechanical stretching and thinning of the choroid and RPE. [14], [15]. Changes in peripheral retina of myopic are predisposing factors for retinal detachment and include lattice degeneration, white-without-pressure, pigmentary degenerations, and retinal tears and holes. Association of extensive myelinated nerve fibers and high degree myopia have been reported [16]. Ellis et al [17] found that $83 \%$ of patients with myelinated retinal nerve fibers had myopia greater than 6 diopters. It is not clear whether myelination of retinal nerve fibers is the reason for or the result of myopia. In patient with myelinated fibers retinal images may be blurred causing visual deprivation. This deprivation may contribute to myopia by including an axial enlargement. On the other hand, it is also possible that axial elongation predisposes to retinal nerve fiber myelination. Straatsma et al [18] found that $10 \%$ of patients with myelinated nerve fibers have myopia, ampliopia, and strabismus. Our patient had refractive error of -9.0 in OD and -5.5 in OS. In pathologic myopia progressive chorioretinal degeneration is often associated [19]. In our patient's OS where MRNF lesions were found posterior cortical vitreous partially separated from the retina (epiretinal membrane) and some tractional areas remain adherent to portions of the macula causing Vitreomacular Traction Syndrome (VTS).

\section{Compliance with ethical standards}

Conflict of interest: The authors declare that they have no conflict of interest. 
Ethical approval: All procedures performed in studies involving human participants were in accordance with the ethical standards of the institutional and/or national research committee and with the 1964 Helsinki Declaration and its later amendments or comparable ethical standards

Informed consent: Informed consent was obtained from patient's parents to publish his photo and all other information

\section{References}

1. Straatsma BR, Foos RY, Heckenlively JR, Taylor GN. (1981) Myelinated retinal nerve fibers. Am J Ophthalmol. 91: 25-38.

2. Kodama T, Hayasaka S, Setogawa T. (1990) Myelinated retinal nerve fibers: Prevalence, location and effect on visual acuity. Ophthalmologica. 200: 77-83. [Crossref]

3. Serdar O, Mehmet YT. (2017) Ring-shaped myelinated retinal nerve fibers at fovea. Indian J Ophthalmol. 65(7): 630-632. [Crossref]

4. Darin RG. (2018) Atlas of Retinal OCT. Optical Coherence Tomography 2018, Elsevier;2018

5. Prakalapakorn SG, Buckley EG. (2012) Acquired bilateral myelinated retinal nerve fibers after unilateral optic nerve sheath fenestration in a child with idiopathic intracranial hypertension. J Pediatr Ophthalmol Strabismus. 16: 534-8. [Crossref]

6. Bradley R. Straatsma, John R. Heckenlively, Robert Y. Foos, and John K. (1979) Shahinian, Myelinated retinal nerve fibers with ipsilateral myopia, amblyopia, and strabismus. American Jopurnal of Opthalmology. 88: 506-510. [Crossref]

7. Smitdt D, Meyer JH, Brandi-Dohrn J. (1996-1997) Wide-spread myelinated nerve fibers of the optic disc: do they influence development of myopia? Int Ophthalmol. 20(5): 263-8. [Crossref]

8. Hollo G. (2016) Infuence of myelinated retinal nerve fibers on retinal vessels density measurement with AngioVue OCT angiography. Int Ophthalmol. 36(6): 915-919. [Crossref]

9. Sowkia JW, Nadeau MJ. (2013) Regresion of myelinated nerve fibers in a glaucomatous eye. Optom Vis Sci. 90(7): e2018-e220. [Crossref]

10. Chavis PS, Tabbara KF. (1998) Demyelinization of retinal myelinated nerve fibers in Behcet's disease. Doc Opthalmol. 95: 157-64. [Crossref]

11. Hayashi K, Ohno-Matsui K, Shimada N, Moriyama M,Kojima A, Hayashi W, Yasuzumi K, Nagaoka N, Saka N, Yoshida T, Tokoro T, Mochizuki M. (2010) Long-term pattern of progression of myopic maculopathy: a natural history study. Ophthalmology 117: 1595-611. [Crossref]

12. Pan CW, Zheng YF, Wrong TY, et al. (2012) Variation in prevalence of myopia between generations of migrant Indians living in Singapore. Am J Ophthalmol 154: 376-81. [Crossref]

13. Baker BJ, Pruett R. (2004) Degenerative myopia. In: Yanoff M, Duker SJ. Ophthalmology: $2^{\text {nd }}$ ed. Spain : Mosby 934-7.

14. Grossniklaus HE, Green WR. (1992) Pathologic findings in pathologic myopia: Retina 12(2): 127-133. [Crossref]

15. Rabb MF, Garoon I, LaFranco FP. (1981) Myopic macular degeneration. Int Ophthalmol Clin 21(3): 51-69. [Crossref]

16. Elvan Yalcın, Ozlem Balc1, and Ziya Akıngol(2013 ) Indian J Ophthalmol. 61(10): 606-607;

17. Ellis GS Jr, Frey T, Gouterman RZ. (1987) Myelinated nerve fibers, axial myopia, and refractory amblyopia: An organic disease. J Pediatr Ophthalmol Strabismus 24: 111-9. [Crossref]

18. Straatsma BR, Heckenlively JR, Foos RY, Shahinian JK. (1979) Myelinated retinal nerve fibers associated with ipsilateral myopia, amblyopia, and strabismus. Am J Ophthalmol 88: 506-10). [Crossref]

19. Soubrane G, Coscas G.J. (2001) Choroidal neovascularization in degenerative myopia. In: Ryan SJed. Retina. $3^{\text {rd }}$ St Louis, Mo Mosby Inc 1136-1152.

\section{Citation:}

Jovanovic Dragan, Ruszkowski Alissa (2019) Myelinated nerve fiber layer associated with other ocular pathology in a 20-year-old myopic man. J Clin Res Med Volume 2(3): $1-3$. 